\title{
URBAN LAND COVER CLASSIFICATION USING HYPERSPECTRAL DATA
}

\author{
Gaurav Hegde ${ }^{\mathrm{a}}$, J. Mohammed Ahamed ${ }^{\mathrm{b}, *}$, R. Hebbar $^{\mathrm{b}}$ and Uday Raj $^{\mathrm{b}}$ \\ ${ }^{a}$ M.Tech Student, National Institute of Technology (NITK), Suratkal, Karnataka, India - (gauravhegde24@gmail.com) \\ ${ }^{\mathrm{b}}$ Regional Remote Sensing Centre-South, NRSC/ISRO, ISITE Campus, Bangalore, India - \\ (mohammedahamed_j, hebbar_kr, udayraj)@nrsc.gov.in
}

KEY WORDS: Hyperion, MNF, Urban, Land cover, Spectral Angle Mapper, Support Vector Machine

\begin{abstract}
:
Urban land cover classification using remote sensing data is quite challenging due to spectrally and spatially complex urban features. The present study describes the potential use of hyperspectral data for urban land cover classification and its comparison with multispectral data. EO-1 Hyperion data of October 05, 2012 covering parts of Bengaluru city was analyzed for land cover classification. The hyperspectral data was initially corrected for atmospheric effects using MODTRAN based FLAASH module and Minimum Noise Fraction (MNF) transformation was applied to reduce data dimensionality. The threshold Eigen value of 1.76 in VNIR region and 1.68 in the SWIR region was used for selection of 145 stable bands. Advanced per pixel classifiers viz., Spectral Angle Mapper (SAM) and Support Vector Machine (SVM) were used for general urban land cover classification. Accuracy assessment of the classified data revealed that SVM was quite superior (82.4 per cent) for urban land cover classification as compared to SAM (67.1 per cent). Selecting training samples using end members significantly improved the classification accuracy by 20.1 per cent in SVM. The land cover classification using multispectral LISS-III data using SVM showed lower accuracy mainly due to limitation of spectral resolution. The study indicated the requirement of additional narrow bands for achieving reasonable classification accuracy of urban land cover. Future research is focused on generating hyperspectral library for different urban features.
\end{abstract}

\section{INTRODUCTION}

Information on urban land cover types and their spatial distributions is essential for a wide range of studies in social sciences, demographics as well as by municipalities for developmental plans and decision making process (Stefanov et al., 2001). It is an essential element for modelling and understanding the earth as a system (Lillesand et al., 2009). Land cover is a driver of climate change and is a central input to both global and regional scale climate models (Vargo et al., 2013). Particularly in urban environments, fast changing land cover patterns play key role in changing local weather under current climate change scenarios.

Multispectral satellite datasets have been commonly used for remotely sensed classification of land use and land cover (Jensen, 1996). Land use and land cover classification accuracy in urban areas is limited owing to the broad spectral resolution of multispectral satellite data and spatial heterogeneity. However, advances in imaging spectrometers have begun to fill the gap in multispectral sensor limitations (Heiden et al., 2007).

Accurate classification of land cover and land use classes in the complex urban environment using satellite remote sensing is a challenging task. The multispectral data have been used extensively for mapping urban land cover classification and analysing the urban sprawl dynamics (Jusoff and Hassan., 1998, Sudhira et al., 2004 and Anurag Ori and Poonam, 2012). Spectral mixing is one of limitation of multispectral data for accurate classification of urban land cover. In this context, hyperspectral data enable more accurate discrimination of urban features (Tan and Wang, 2007). In addition to classifying whole image into a set of classes, presence of numerous bands enable detailed feature identification and characterization of surface materials (Bokoye, and Dionne, 2004 and Heiden et al., 2007).
The urban land cover is characterised by heterogeneous combination classes such as water bodies, recreational parks, vegetation, residential, industrial and open lands etc. Accurate classification of urban land cover is prerequisite for studying long term dynamics of urbanization and its impact on urban ecosystem. Bengaluru city has seen unprecedented urban growth during last decade and studies have demonstrated the potential use of multi-spectral RS data for analysing of urban growth dynamics of the city (Ramachandra and Uttam Kumar, 2009). The spatial and spectral resolution of medium resolution data limits the accurate classification of urban land cover to achieve reasonable accuracy. In this context, hyperspectral data provides adequate spectral resolution data for improved classification of urban land cover mapping.

Traditionally several algorithms are available to classify multispectral data such as Gaussian Maximum Likelihood Classifier (GMLC), Minimum distance classifier, Mahanalobis distance classifier etc. GMLC is the most common algorithm used in multispectral image classification. However, its application for classification hyperspectral data is still limited due high dimensionality and redundancy of hyperspectral data and thus, the data mining is essential (Thenkabail et al., 2011). The advanced and efficient classification algorithms such as Spectral Angle Mapper (SAM) and Support Vector Machine (SVM) have been developed to analyse hyperspectral data. The present study was carried out to study the potential use of Hyperion data for selection of optimal bands and delineation of urban land cover using advanced classifiers such as SAM and SVM.

\footnotetext{
* Corresponding author.
} 


\section{STUDY AREA AND MATERIALS}

\subsection{Study Area}

Bengaluru city, IT capitol of Karnataka and sixth largest metropolis in India, was selected as study area. Geographic extent of the study area ranges from $13^{\circ} 27^{\prime} 12.20^{\prime \prime} \mathrm{N}, 77^{\circ} 47^{\prime}$ $31.64 "$ to $12^{\circ} 35^{\prime} 10.59^{\prime \prime} \mathrm{N}, 77^{\circ} 40^{\prime} 51.56$ " E. Located at an average elevation of about $900 \mathrm{~m}$ in the Deccan plateau. Study area consists of dense built up areas, vegetation, croplands and numerous tanks making it an ideal urban landscape.

\subsection{Datasets}

Hyperion data acquired over the test site on October, 052012 by Hyperion (Path/Row No: 144/51). The imagery was received as a full scene strip, ortho-rectified in GEOTIFF format, with information as radiance values. Image processing software viz., ENVI and ERDAS was used for satellite data analysis and accuracy assessment.

\section{METHODOLOGY}

The methodology included standard hyperspectral data processing and analysis techniques as presented in Figure-1. Hyperion data was initially converted to radiance by multiplying the appropriate factor provided along with data followed by atmospheric correction. Later with the noise segregated image, classification procedures were carried out using advanced pixel based classifiers SAM and SVM algorithms for deriving land cover map. The accuracy assessment of classified map was carried out by comparing with high resolution data.

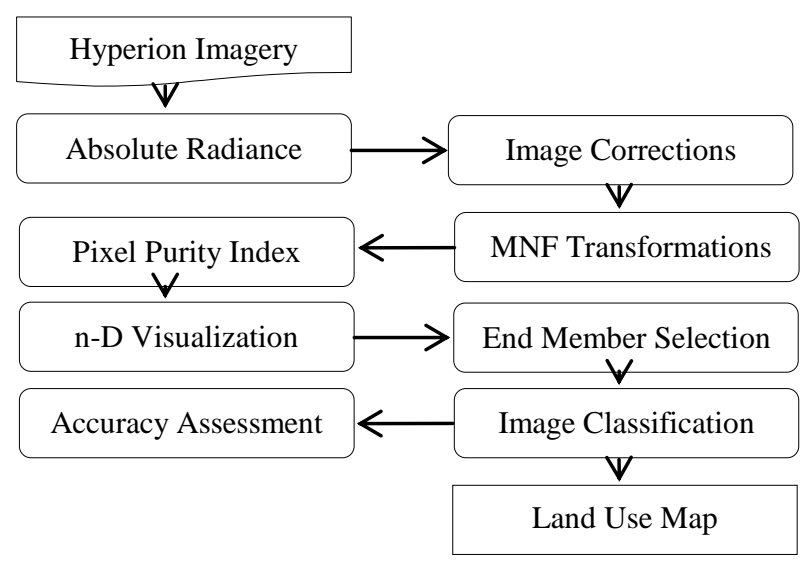

Figure-1: Methodology adopted in the study

\subsection{Spectral Resizing and Image Correction}

Hyperion imagery with 242 bands was layer stacked and atmospheric correction was applied using Fast Line-of-sight Atmosphere Analysis of Spectral Hypercube (FLAASH) as adopted in the ENVI package. Subsequently the non-calibrated bands, water absorption bands and additional bands with streaks / stripes were removed based on visual inspection of the data.

\subsection{Minimum Noise Fraction Transformation}

Minimum Noise Fraction (MNF) transformation was applied on the atmospherically corrected image to reduce data dimensionality. In order to segregate the better quality bands, coherent bands with higher Eigen values were selected by eliminating redundant bands and bands containing erroneous information. In the present study, MNF for VNIR and SWIR bands were applied separately as they have different information and noise content. Inverse MNF transformation was applied to obtain about 145 spectral bands which were noise segregated and used for further data analysis.

\subsection{End Member Selection}

End member selection was carried out directly from inverse MNF image, Pixel Purity Index (PPI) image and scatterplots. In this study both methods were followed to study the effect of refining endmember pixels using n-D visualizer on accuracy of classification.

PPI technique helps to determine the most spectrally pure pixels in a multispectral or hyperspectral domain in which the digital number (DN) of each pixel corresponds to the number of times same pixel was judged as extreme (Singh et al., 2013). PPI transformation was applied on a MNF transformed image. In the PPI output image, the brighter pixels represented pure pixels and more spectrally extreme pixels. Darker pixels represented pixels with mixed features and were less spectrally pure. n-D visualizer was used to identify, locate and cluster the purest pixels belonging to a particular endmember (Ahmad, 2012) and each spectra was represented as points with bands as axis of the scatterplots. Resulting PPI image was used in scatterplots and $\mathrm{n}-\mathrm{D}$ visualizer for creating Region of Interest (ROI) for image classification. Typical and representative 2D scatterplots of end member spectra is depicted in Figure-2.
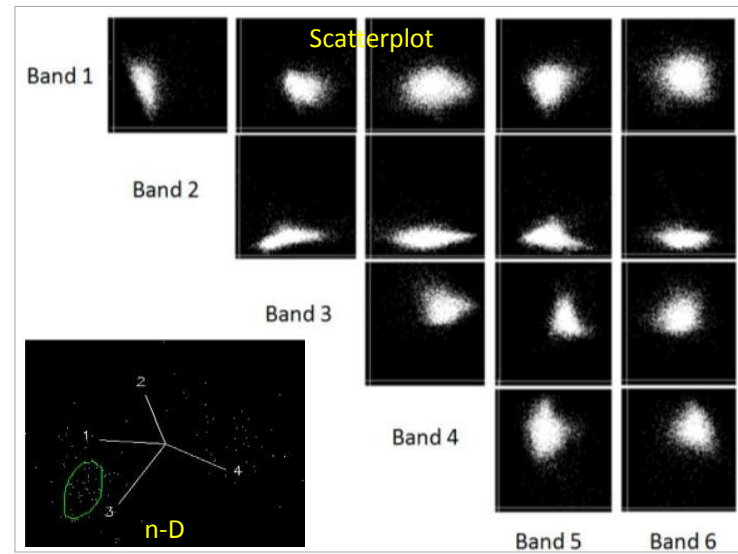

Figure-2: n-D Visualizer interface for refining the reference endmember ROIs selected from scatterplots.

\subsection{Image classification and accuracy assessment}

The training samples, selected directly from the image as well as using $n-D$ visualizer were used for classification employing SAM and SVM algorithm. Typical representative sample sites were identified using high resolution data covering seven major land cover classes existing in the study area. In the present study, homogeneous, spatially distinct with reference to Hyperion spatial resolution and independent sample sets were identified from high resolution LISS-IV + Cartosat-1 merged data of $2.5 \mathrm{~m}$ for classification. Accuracy assessment was carried out to compare the efficacy of refining training samples using end member spectra by generating confusion matrix along with general accuracy statistics (Congalton, 1991). 


\section{RESULTS AND DISCUSSION}

The MNF transformed images were analyzed for elimination of redundant bands and segregate noise to increase the computational efficiency of subsequent processes (Satpathy et al., 2010). This resulted in 13 MNF bands in the VNIR region with Eigen value up to $1.76(84.0 \%)$ and $50 \mathrm{MNF}$ bands in SWIR region with Eigen value up to 1.67 (80.9\%). The inverse MNF transformation of the above bands resulted in 145 stable bands. Figure-3 shows eight best MNF bands in the in the VNIR in decreasing order of Eigen values. The results indicated the importance of SWIR bands for identification of urban features.

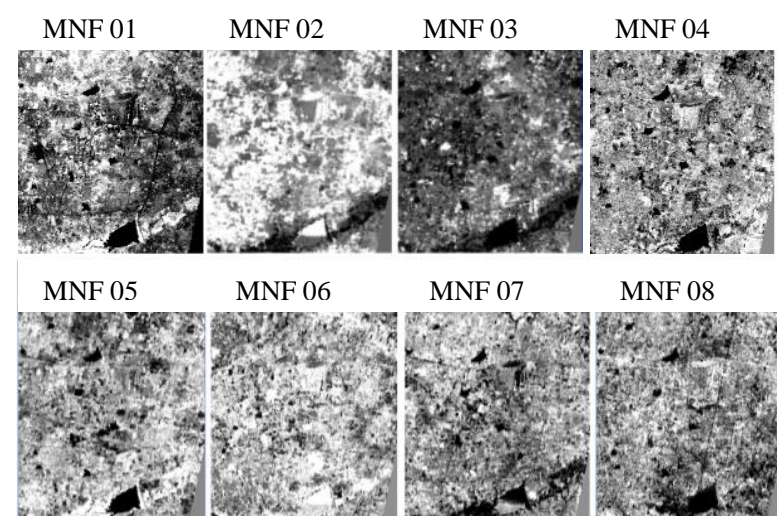

Figure-3: MNF bands of VNIR region

The hyperspectral profile for seven major urban land cover classes was generated to analyze the class separability and selection of bands that are best suitable for improved discrimination built-up classes with in the urban area. The overall spectral reflectance varied for different land cover classes and the largest dynamic range in the SWIR region. Higher spectral reflectance was observed for sheet roof in the entire 400-2500 spectrum region. Similar patterns were also observed between shallow water and built up class. Among the different urban features, asphalt was spectrally distinct from built up and roof sheets. However, asphalt and built up class was spectrally similar in the VNIR regions and separable in the SWIR bands. Vegetation class was spectrally distinct from other land cover classes; however, emphasis was not given to distinguish different vegetation types (Figure-4). Similar spectral reflectance trends were observed by Martin et al., 2002.

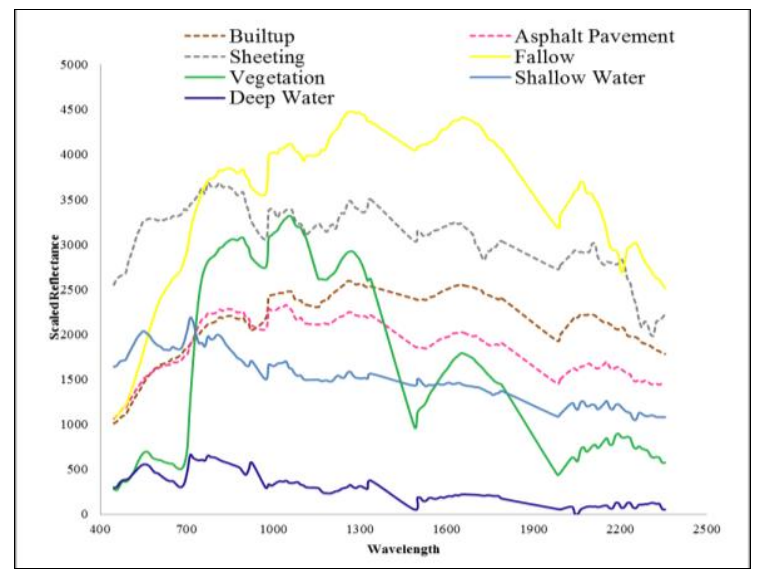

Figure-4: Spectral reflectance curves of various endmember classes obtained from image
The general urban land cover map generated from Hyperion data using SAM and SVM classification technique is depicted in Figure-6. The SAM classifier showed poor classification in comparison to SVM in spatially depicting different land cover classes of the study area. SAM resulted in large number of

unclassified pixels. In contrast to this, SVM was able to classify major urban land cover classes such as water, built up and vegetation. Two major features within the urban class i.e., asphalt and built up features were more accurately classified compared to SAM. Similarly, shallow and deep water bodies could be delineated. SVM classification using training samples selected from end member spectra has shown best results as depicted in Fifure-5c.

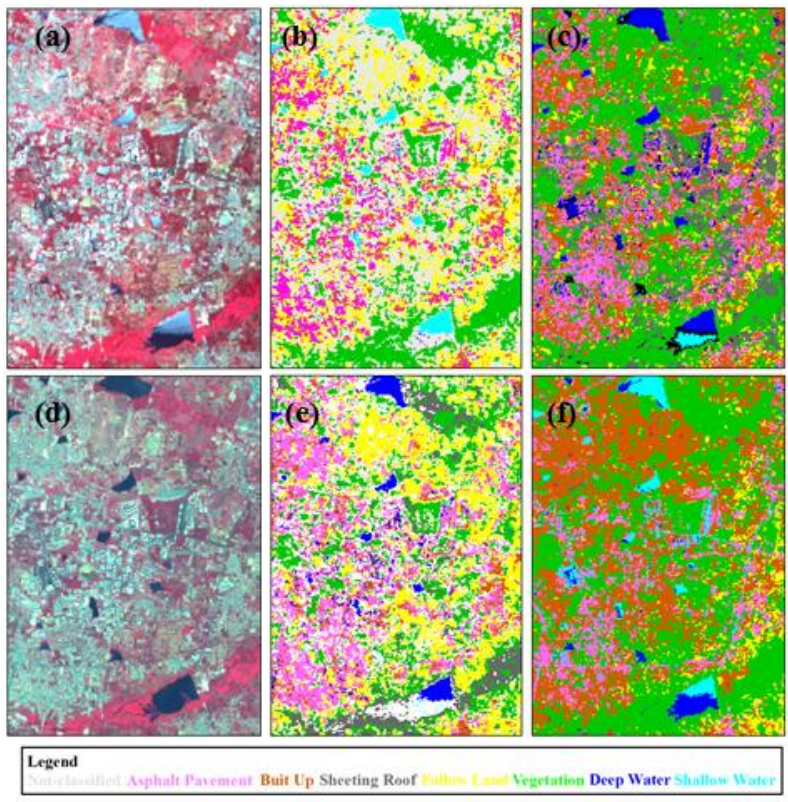

Figure-5: (a) Hyperion FCC (b) LISS-III FCC (c) SAM Classified image with training samples from MNF scatterplots (d) SAM Classified image with training samples taken from image (e) SVM Classified image with training samples from MNF scatterplots (f) SVM Classified image with training samples directly from image

Table-1 confirms the superiority of the SVM in terms of both overall accuracy and Kappa coefficient in comparison to SAM. The higher classification accuracy reported by SVMs is mainly attributed to the fact that this classifier has been designed identify an optimal hyper plane for class separation (George el al., 2012). The limitation of SAM for land cover classification is large unclassified pixels within the spectral angle threshold of 0.12 radians. Lower accuracy of SAM could also be due to higher variation in reflectance values of classes. Better performance of SVM is also due to its ability to work with smaller training samples.

Reference pixels selection from the image or end member spectra showed marked difference in classification accuracy. The training sample selection directly from the image resulted in poor overall classification accuracy in both SAM (63.4\%) and SVM $(68.6 \%)$. It is evident from the results that refining end member pixels using scatterplots and n-D visualizer improved classification accuracy. However, improvement in 
accuracy was quite significant in SVM $(20.1 \%)$ as compared to SAM classifier $(5.9 \%)$. This is primarily due to unbiased training sample selection through the end member spectra. SVM algorithm which is a binary classifier was able to distinguish spectrally similar classes like asphalt pavement and built up class using training samples from end members spectra unlike SAM which led to misclassification in these classes.

Table-1: Classification performance of SAM and SVM

\begin{tabular}{|r|c|c|}
\hline & \multicolumn{2}{|c|}{ Overall Accuracy (\%) } \\
\hline & SAM & SVM \\
\hline 1. Samples from image \\
\hline Overall & 63.4 & 68.6 \\
\hline Kappa & 0.61 & 0.65 \\
\hline 2. Samples from n-D Visualizer \\
\hline Overall & 67.1 & 82.4 \\
\hline Kappa & 0.62 & 0.79 \\
\hline
\end{tabular}

\section{CONCLUSIONS}

The present study was carried out to explore abundant spectral information available in Hyperion data for urban land cover classification. The pre-processing of hyperspectral data included atmospheric correction, MNF transformation and generation of PPI images. About 145 stable bands were used in the final analysis. Two advanced classifiers SAM and SVM was used for classification of the data using training sample obtained from the image as well as using scatterplots and n-D visualizer. The results indicated that SVM classification showed consistent results and higher classification accuracy which indicated its better performance for urban classification. SAM resulted in poor classification accuracy and large no. of unclassified pixels.

The present study explored the advantage of hyperspectral data for urban studies utilising the higher spectral resolution. The study indicated the potential of Hyperion data for discrimination of three classes within the built up area using SVM algorithm. However, further study is essential to explore both high spatial and spectral resolution data for improved discrimination of compact urban land use and land cover classification.

\section{REFERENCES}

Ahamad F., 2012, Pixel Purity Index Algorithm and nDimensional Visualization for ETM+ Image Analysis: A Case of District Vehari. Global Journal of Human Social Science Arts \& Humanities. 12 (15): 76-82.

Anurag Ori and Poonam, 2012, Urban sprawl mapping and land use change detection using remote sensing \& GIS. International Journal of Remote Sensing and GIS, 1 (1), pp:12-25.

Bokoye, A.I. Dionne, P. 2004, Urban material characterization from the Hyperion hyperspectral imager, Application to downtown Montreal (Quebec, Canada). Image and signal processing for remote sensing. 5238, pp: 569-574.

Congalton, R. G., 1991, A review of assessing the accuracy of classifications of remotely sensed data. Remote Sensing of Environment, 37, pp: 35- 46.
George, P. P., Arvanitis, K. and Sigrimis, N., 2012, Hyperion hyperspectral imagery analysis combined with machine learning classifiers for land use/cover mapping. Expert Systems with Applications, 39, pp: 3800-3809

Heiden, U., Segl K. Roessner, S. and Kaufmann, H., 2007, Determination of robust spectral features for identification of urban surface materials in hyperspectral remote sensing data. Remote Sensing of Environment, 111, 537-552.

Jensen, J.R., 1996. Introductory digital image processing: A remote sensing perspective. Prentice-Hall, Upper Saddle River.

Jusoff, K. and Hassan, H. M., 1998. An overview of satellite remote sensing for land use planning with special emphasis in Malaysia. Remote Sensing Reviews, Vol 16(3), 209-231

Lillesand TM, Kiefer RW and Jonathan, C.W., 2009, Remote sensing and image interpretation, 4th Edition, John Wiley \& Sons, Inc., New York.

Martin, H., Meg, G., Brian, H. and Roberts, D., 2002, The spectral dimension in urban land cover mapping from high resolution optical remote sensing data. Proceedings of the 3rd Symposium on Remote Sensing of Urban Areas, Istanbul, Turkey.

Ramachandra, T.V. and Uttam Kumar, 2009, Geoinformatics for urbanization and urban sprawl pattern analysis. In Geoinformatics for Natural Resources Management. 426-471.

Satpathy, R., Singh, V. K., Parveen, R., and Jeyaseelan, A. T., 2010. Spectral Analysis of Hyperion Data for Mapping the Spatial Variation of in a Part of Latehar \& Gumla District, Jharkhand. Journal of Geographic Information System. 2, 210216.

Singh, K., Rajaprian, K., Vinothkumar, M. and Kumar, R. S., 2013. Hyperspectral remote sensing approach for lithological discrimination by ASTER Data-A Case Study of Thenkalmalai and Odhimalai Hills, Tamilnadu, India. International Journal of Advanced Earth Science and Engineering, 2(1), pp:75-79.

Sudhira, H.S. Ramachandra T.V. and Jagadish, K.S., 2004, Urban sprawl: metrics, dynamics and modelling using GIS. International Journal of Applied Earth Observation and Geoinformation 5, pp:29-39.

Tan, Q. and Wang, J., 200, Hyperspectral Versus Multispectral Satellite Data For Urban Land Cover And Land Use MappingBeijing, An Evolving City, ASPRS 2007 Annual Conference. Thenkabail P. S., Lyon J. G. and Huete A., 2011, Hyperspectral Remote Sensing of Vegetation. pp. 94-97.

Vargo, J., Habeeb, D. and Stone Jr, B., 2013, The importance of land cover change across urban-rural typologies for climate modeling. Journal of environmental management, 114, 243-252. 